Elsevier required licence: (C) $<2016>$. This manuscript version is made available under the CC-BY-NC-ND 4.0 license http://creativecommons.org/licenses/by-nc-nd/4.0/ 


\title{
The structure and intensity of self-reported autonomic arousal symptoms across anxiety disorders and obsessive-compulsive disorder
}

\author{
3685 words (Excl. references)
}

David Berle ${ }^{1,2}$, Vladan Starcevic ${ }^{3}$, Denise Milicevic ${ }^{4}$, Anthony Hannan ${ }^{4}$, Erin Dale ${ }^{4}$, Brian Skepper ${ }^{4}$, Kirupamani Viswasam ${ }^{5} \&$ Vlasios Brakoulias ${ }^{3}$.

1. School of Psychiatry, UNSW Australia.

2. St John of God Health Care, Richmond Hospital, Australia.

3. Discipline of Psychiatry, Sydney Medical School - Nepean, University of Sydney, Australia.

4. Nepean Anxiety Disorders Clinic, Nepean Blue Mountains Local Health District, Australia.

5. Department of Psychiatry, Nepean Hospital, Penrith, Australia.

Address for correspondence:

David Berle

School of Psychiatry, UNSW Australia

Black Dog Institute Building

Hospital Road, Prince of Wales Hospital,

Randwick NSW 2031

Australia

Email: d.berle@unsw.edu.au

$\mathrm{Ph}:+61293828503$

Fax: +61 293828151 


\begin{abstract}
Background: Heightened autonomic arousal symptoms (AAS) are assumed to be a central feature of anxiety disorders. However, it is unclear whether the magnitude and profile of AAS vary across anxiety disorders and whether heightened AAS characterises obsessive-compulsive disorder (OCD). Aims: We sought to determine whether the intensity and structure of AAS varied across anxiety disorders and OCD. Method: A sample of 459 individuals with a primary anxiety disorder or OCD were administered the Symptom Checklist-90R. Nine items referring to prototypic AAS were included in a latent class analysis. Results: A 2-class solution (high and low AAS classes) best fitted the data. Participants comprising the high AAS class scored uniformly high across all assessed AAS symptoms. Older age and the presence of panic disorder, social anxiety disorder and generalized anxiety disorder predicted membership in the high AAS class. No OCD symptom dimension was significantly associated with membership in the high AAS class. Limitation: AAS were assessed using a self-report measure and replication is needed using other methodologies. Conclusions: These findings suggest that OCD may be sufficiently distinct from anxiety disorders and do not support subtyping of anxiety disorders on the basis of the predominant type of AAS. Therapeutic approaches that target AAS might best be applied in the treatment of panic disorder, social anxiety disorder and generalized anxiety disorder.
\end{abstract}

Keywords: Autonomic arousal; sympathetic nervous system; anxiety disorders; obsessive-compulsive disorder; fear. 
There is an increasing appreciation of the diversity of anxiety disorders. Anxiety disorders not only vary in terms of the feared object or situation, but also in the patterns of prototypic responses to perceived threats (e.g., avoidance). Autonomic arousal (AA) is assumed to be one of the few unifying features of anxiety disorders, as proposed by models that delineate anxiety disorders from depression (e.g., the Tripartite model; Clark \& Watson, 1991) and consistent with the assumption that fear characterises all anxiety disorders (American Psychiatric Association, 2013). However, it remains unclear whether there is variation in either the structure or severity of AAS between individuals with various anxiety disorders.

So far as the structure of autonomic nervous system symptoms is concerned, there may be variation in symptom profiles between different groups of individuals with anxiety disorders. This would be evident if certain AAS were more prominent in some groups of individuals than others such that these symptoms might be grouped in meaningful ways that correspond to particular groups of individuals with anxiety disorders. For instance, a respiratory subtype was identified in individuals with panic disorder (Briggs et al., 1993; Roberson-Nay et al., 2012). This subtype comprises symptoms of dyspnoea, chest pain, feelings of choking and paraesthesia, in contrast to a non-respiratory cluster of symptoms including tachycardia, sweating and trembling. However, beyond respiratory-challenge and biological studies that have supported such a distinction, the evidence otherwise remains inconclusive (Freire et al., 2010) and the degree to which a subtype of respiratory-prominent AAS extends to other (non-panic) anxiety disorders is unclear. In social anxiety disorder, there is preliminary evidence that individuals who report prominent blushing may also be characterised by elevated heart rates (and palpitations) during exposure to social stressors (Gerlach et al., 2001) and that they are distinguishable from others with social anxiety disorder. Again, it is unclear to what extent such a cluster of symptoms extends beyond social anxiety disorder. Improving our understanding of the ways in which AAS cluster may provide insights for targeted treatment approaches (e.g., the use of controlled breathing strategies for individuals with prominent respiratory symptoms). 
Individuals with anxiety disorders may also differ in the overall severity of their autonomic symptoms, regardless of how individual symptoms cluster together. In this respect, there may be between-disorder differences in the magnitude of all AAS, regardless of their type. Attempts have been made to distinguish "fear-circuitry" disorders, such as panic disorder, social anxiety disorder and specific phobias, from "anxious-misery" disorders, including generalized anxiety disorder (GAD) and depression (Andrews, Charney, \& Sirovatka, 2009) - with posttraumatic stress disorder (PTSD) potentially having features of each (Forbes et al., 2011). A similar dichotomy between fear-related and distress disorders has been proposed by Watson (2005). A key difference between these groups of disorders is thought to be the magnitude of AAS, with the fear-circuitry disorders characterised by greater levels of autonomic arousal.

There is some support for the notion that disorders may be distinguishable by overall levels of autonomic arousal. Brown and McNiff (2009) investigated autonomic arousal in 293 outpatients attending an anxiety disorders centre. Self-reported AAS were associated with panic disorder and PTSD, but not with GAD, obsessive-compulsive disorder (OCD) or social anxiety disorder. These findings were thus broadly consistent with the notion that fear-circuitry disorders might be characterised by more prominent AAS, social anxiety disorder notwithstanding.

The recent release of the Fifth Revision of the Diagnostic and Statistical Manual of Mental Disorders (DSM-5; American Psychiatric Association, 2013) signalled a shifting conceptualisation such that PTSD and OCD are now considered to be key members of the trauma- and stressor-related and obsessive-compulsive and related disorders, respectively. The DSM-5 criteria for PTSD explicitly acknowledge a broad range of emotions (including horror, anger, guilt and shame), besides fear. While AAS are not a part of the official diagnostic criteria for PTSD, a cluster of symptoms labelled "marked alterations in arousal" remains essential for the DSM-5 diagnosis of PTSD. With regards to $O C D$, the nature and severity of AAS associated with this condition remains less well researched and less clear. On the one hand, some studies suggest high levels of fear (and by extension, high levels of AA; Prenoveau et al., 2010) in OCD. However, other studies have suggested relatively low levels of 
autonomic arousal (Pruneti et al., 2010) in OCD, and levels of AAS may be determined in part by the predominant $O C D$ symptom subtype. For instance, individuals with $O C D$ and "not just right" feelings and associated compulsions may report guilt and emotional states that are unrelated to AAS (Mancini et al., 2008).

A recent study found that all OCD symptom dimensions were related to fear (Raines et al., 2015). Contamination obsessions and washing compulsions were exclusively related to fear, whereas other OCD symptom dimensions were additionally related to distress. However, the authors of this study relied on the Panic Disorder Severity Scale (PDSS; Shear et al., 1997) for the measurement of fear symptoms and the Penn State Worry Questionnaire (PSWQ; Meyer et al., 1990) for the measurement of distress symptoms. The PDSS includes items related to distress (associated with panic attacks) as well as fear and the PSWQ is confined to measuring distress in the context of worry. Moreover, the sample was recruited online and included individuals who did not necessarily meet criteria for OCD. There is thus a need for a more rigorous assessment of AAS in an OCD sample.

The present study had two aims. First, we wanted to determine whether there are distinct profiles of AAS in anxiety disorders and OCD such that particular groups of individuals with these disorders might be characterised by distinct clusters of AAS. To the best of our knowledge, such a comprehensive transdiagnostic investigation of AAS has not been conducted previously. In this regard, we expected that respiratory symptoms (dyspnoea, chest pain, paraesthesia) might cluster separately from other autonomic symptoms and that they might especially characterise individuals with panic disorder. Second, we compared various anxiety disorders and OCD in terms of the severity of AAS, expecting prototypic fear-circuitry disorders, such as panic disorder and social anxiety disorder, to be characterised by more severe AAS (regardless of type) than GAD. With regard to $O C D$, the absence of previous studies addressing this question in clinical samples and equivocal findings from the aforementioned literature precluded definitive hypotheses, although the findings of Raines et al. (2015) suggested that perhaps all OCD symptom dimensions might be associated with fear and by extension, with AAS. 


\section{Method}

\section{Participants}

A total of 459 participants (mean age $=40.21 S D=14.41 ; n=146[31.8 \%]$ males) were recruited through the Nepean Anxiety Disorders Clinic and the Department of Psychiatry at Nepean Hospital. All participants were on a waiting list before attending either of these outpatient services and those taking medications were on a stable pharmacotherapy regimen. Two-hundred and forty one (52.5\%) were married or in a de facto relationship, 211 (46.0\%) were currently engaged in paid employment and 69 (15.0\%) had completed a bachelor's degree or higher level of education.

Approval for the study was obtained from the Nepean Blue Mountains Local Health District Human Research Ethics Committee (study numbers 04/053 \& 07/032) and all participants provided written signed consent before the questionnaires were administered.

Participants had the following DSM-IV diagnoses, where a "primary disorder" refers to the condition which was causing them the most distress and impairment: panic disorder with/without agoraphobia $n=195$ (42.5\%; primary diagnosis $n=140,30.5 \%$ ); social anxiety disorder $n=106$ (23.1\%; primary diagnosis $n=42,9.2 \%$ ), GAD $n=175$ (38.1\%; primary diagnosis $n=57,12.4 \%$ ), OCD $n=225$ (49.0\%; primary diagnosis $n=213,46.4 \%)$, specific phobia $n=96$ (20.9\%; primary diagnosis $n$ $=4 ; 0.9 \%)$ and anxiety disorder not otherwise specified $n=3(0.7 \%$; primary diagnosis $n=3,0.7 \%)$. Overall, 317 participants (69.1\%) had at least one co-occurring Axis I diagnosis, and 187 (40.7\%) had two or more co-occurring Axis I diagnoses.

\section{Measures}

Participant diagnostic groupings were derived from the clinician-rated Mini Neuropsychiatric Interview (MINI; Sheehan et al., 1999). The MINI has been validated against other widely used structured and semi-structured diagnostic interviews and has been shown to have good psychometric properties. All research staff were trained in the use of the MINI and interrater 
reliability data were obtained for 48 of the interviews. Kappa values of interrater agreement were 0.93 for panic disorder, 0.89 for social anxiety disorder, 0.82 for GAD and 1.00 for OCD.

Symptoms of autonomic arousal were derived from the Symptom Checklist 90-Revised (SCL90R, Derogatis, 1994). Each item of the SCL-90R is rated on a 5-point scale ranging from 0 ("Not at all") to 4 ("Extremely"). In the absence of a specific inventory of AAS, the SCL-90R assessed 9 items that broadly pertained to autonomic nervous system arousal. The items were 1 . Faintness or dizziness, 2. Pains in the heart or chest, 3. Trembling, 4. Heart pounding or racing, 5. Nausea or upset stomach, 6. Trouble getting your breath, 7. Hot or cold spells, 8. Numbness or tingling in parts of your body, and 9. A lump in your throat. While some of these items could reflect non-autonomic nervous system processes (e.g., a lump in your throat), we included all items that could potentially be relevant on the basis that the study was exploratory and that overinclusion had a relatively lower risk of compromising the study's objectives than omission of the relevant items. The SCL-90R instructs respondents to rate how much each respective symptom has bothered them during the past week. Thus, participant ratings reflect the average symptom activation and interference, rather than an absolute maximum.

The Vancouver Obsessive Compulsive Inventory (VOCl; Thordarson et al., 2004) was administered to 191 of 213 participants who had a primary diagnosis of $O C D^{1}$. It is a 55 -item scale of OCD symptoms and items are rated on a 5-point Likert-type scale from 0 "Not at all" to 4 "Very Much". There are six separate subscales (contamination, checking, obsessions, hoarding, not just right experiences and indecisiveness). Cronbach $\alpha$ values in the present sample were: $0.94,0.95$, $0.90,0.94,0.89$ and 0.86 for each subscale, respectively. Thordarson et al. (2004) reported that the VOCl had favourable convergent validity ( $r=0.85$ with Padua total score), discriminant validity $(r=$ 0.36 with score on the Eysenck Personality Questionnaire - Revised) and test-retest reliability ( $r=$ 0.97 across 47 days in an OCD sample).

\footnotetext{
${ }^{1}$ Not all participants with a primary OCD were administered the $\mathrm{VOCl}$, as this instrument was added to the study protocol after some participants had already completed the study.
} 


\section{Data analyses}

Descriptive statistics and regression analyses were conducted using SPSS Statistics 22.0 and latent class analyses were conducted using MPlus version 7.31.

Latent class analysis

The Akaike Information Criterion (AIC) and Bayesian Information Criterion (BIC) incremental fit statistics were considered in determining the number of classes. Lower values for AIC and BIC indicate better fit. The Lo-Mendell-Rubin adjusted likelihood ratio test (LMRLRT) allowed comparison of chi-square values for each number of classes, and the entropy value, which reflects the degree of discrimination between classes, is also reported. In addition to these indices, the parsimony of the solution was also considered (Collins \& Lanza, 2010). The number of random starts was set at 1000. Logistic regression analyses

All participants were allocated to their most likely group membership based on the conditional response probabilities for each person. A logistic regression analysis was then conducted to determine whether age, gender and diagnosis predicted membership in the high AAS class. Gender, age, and the presence of key disorders (major depressive disorder, panic disorder, social anxiety disorder, GAD, OCD and specific phobia) were entered into the analysis, regardless of whether these were primary or secondary disorders. Inspection of Variance Inflation Factor (VIF) and Tolerance values indicated that multicollinearity was unlikely to be a problem (all VIF values less than 2.5 and all Tolerance values greater than 0.4).

To allow discussion of our findings with regard to OCD symptom dimensions, we conducted a further regression analysis among the 191 participants who had a primary diagnosis of OCD and who had completed the $\mathrm{VOCl}$. Each subscale of the $\mathrm{VOCl}$ was entered as an independent variable to predict membership of the AAS class. Inspection of VIF and Tolerance values indicated that multicollinearity was unlikely to be a problem (all VIF values less than 3.5 and all Tolerance values greater than 0.25$)$. 


\section{Results}

\section{Latent class analyses}

The summary statistics of the latent class analysis results are summarised in Table 1 . The lowest AIC and BIC values were for a three class solution. The Low-Mendell Rubin test also suggested that 3-classes provided a significantly better fit than the two class solution $(p=0.02)$. However, the entropy value, reflecting classification accuracy, was greatest for the two-class solution (0.91), and inspection of the conditional response probability plots indicated that two of the three classes had highly similar conditional probabilities across all the items. (Additional results for the three class solution are available from the authors upon request). With this in mind and seeking the most parsimonious interpretable solution, we chose the two-class solution.

Conditional response probability plots for the two-class solution at each response level (not at all; a little bit; moderately; quite a bit; extremely) are shown in Figure 1. Clear differentiation between the classes is most evident when symptoms were endorsed at either the level of "not at all" or at the level of "moderate" strength or greater. At these greater levels of intensity, participants had either consistently high or consistently low probabilities of endorsing AAS and participants were not very likely to selectively endorse only some symptoms. The classes were thus labelled "high AAS" (46.5\% of the overall sample) and "low AAS" ( $53.5 \%$ of the sample).

Although the patterns of probabilities were generally consistently high or low in each class, the high AAS class appeared to have a particularly high probability of endorsing "heart pounding or racing" and a relatively low probability (but still higher than the low AAS class) of endorsing "trembling".

\section{Logistic regression analyses}

The results of the logistic regression analysis where disorder and demographic variables predicted class membership are summarized in Table 2 . Older age, as well as the presence of a current panic disorder, social anxiety disorder and GAD (whether primary or secondary) were 
significant independent predictors of membership in the high AAS class. Gender and the presence of OCD, specific phobia and major depressive disorder were not significant predictors of membership in the high AAS class. A separate chi-square analysis suggested that the presence of a co-occurring disorder predicted membership of the high AAS group: $\left(\chi^{2}=28.14, \mathrm{df}=1, \mathrm{p}<0.001\right)$.

Table 3 summarises the results of the logistic regression analysis where each subscale of the $\mathrm{VOCl}$ was used to predict class membership. None of the $\mathrm{VOCl}$ subscales was a significant independent predictor of membership in the high AAS class.

\section{Discussion}

Improving our understanding of the role of autonomic symptoms in anxiety disorders and OCD is important. If autonomic symptoms, such as shortness of breath, are present across all disorders, transdiagnostic treatment approaches might benefit from including de-arousal strategies (e.g., the CALM approach; Sullivan et al., 2007). On the other hand, if AAS (like shortness of breath) only characterise particular disorders, techniques such as breathing retraining, might only be necessary for the treatment of specific disorders (e.g., panic control treatment; Hofmann \& Spiegal, 1999).

Two broad conclusions arise from the results of our study. First, individuals with anxiety disorders appear to either report moderate to strong activation of the AAS that we assessed or no/minimal activation. Second, the presence of panic disorder, social anxiety disorder or GAD is associated with high levels of $A A$, in contrast to specific phobia, $O C D$ and major depressive disorder.

The possibility that AAS in anxiety disorders is an "all-or-nothing" phenomenon is particularly intriguing. To the best of our knowledge, this finding has not been previously reported. It suggests that the threat response system which drives AAS might be relatively "blunt" rather than specific. For instance, if a perceived threat requires increased respiration to facilitate a fight or flight response, other non-essential processes, such as constriction of stomach muscles and associated nausea sensations, may also become activated in the process. Such a general activation of AAS may 
signal to a clinician the presence of an anxiety disorder (our findings for specific phobia notwithstanding), but it does not provide sufficient specificity to assist in differential diagnosis between anxiety disorders.

Ours was the largest study investigating AAS in individuals with various anxiety disorders and OCD. Unlike previous studies, we also examined AAS in individuals with different OCD symptom dimensions. Our findings are only partially consistent with those of Brown and McNiff (2009). We also found that the presence of panic disorder predicted elevated AAS independent of other disorders. This reinforces the notion that panic disorder is a prototypic disorder of AA. However, in contrast to Brown and McNiff, we found that social anxiety disorder and GAD were independent predictors of elevated AAS. For social anxiety disorder, which is thought to often comprise situationally-bound panic attack-like episodes (arising in social contexts; Jack, Heimberg, \& Mennin, 1999), the association with AAS in the present study is not surprising and is consistent with its placement within "fear" (Watson, 2005) and "fear-circuitry" (Andrews et al., 2009) disorder taxonomies. The contrasting findings of Brown and McNiff regarding social anxiety disorder call for replication. The association between GAD and AAS is in contrast to the findings of Brown and McNiff and those of a number of studies that indicate autonomic suppression among individuals with GAD (e.g., Brown et al., 1998). However, there is evidence that large proportions of GAD patients may present with AAS, especially in primary care (Rickels \& Rynn, 2001; Stein et al., 2005). Furthermore, the DSM-IV and DSM-5 conceptualisations of GAD may be "biased" regarding AAS by excluding them from the diagnostic criteria, whereas the International Classification of Diseases regards these symptoms as essential for the diagnosis of GAD (World Health Organisation, 1992).

The presence of a current specific phobia did not predict membership in the high AAS class. Rather than discounting the possibility that specific phobic responses are characterised by AAS, these findings might suggest that AAS is not persistently high for individuals who can easily avoid their feared object or situation (e.g., snakes or heights). In other words, autonomic symptoms in 
such individuals may only become prominent on the infrequent occasions when they are exposed to their feared object.

Membership in the high AAS group was predicted by the presence of a co-occurring Axis I disorder (i.e., major depressive disorder, dysthymia, panic disorder, agoraphobia, social anxiety disorder, OCD, PTSD, GAD or specific phobia). This finding is not unexpected and indicates either that there may a compounding effect of co-occurring disorders so far as AAS are concerned or that if one has a co-occurring disorder, this is likely to be a disorder characterised by high levels of AAS, thus leading to membership in the high AAS class.

Neither OCD, nor any OCD symptom dimensions, served as an independent predictor of membership in the high AAS group. This stands in contrast to the findings of Raines et al. (2015) who reported that all OCD symptom dimensions were associated with fear. Methodological differences may account for this discrepancy in that, to the best of our knowledge, ours is the first study to have investigated this question using a clinical sample of participants with OCD. The apparent lack of association with AAS in the present study might also reflect the complex mix of emotional states implicated in OCD (including disgust and guilt; Berle et al., 2012; Mancini \& Gangemi, 2004).

There are limitations of this study that need to be acknowledged. First, we relied on selfreport ratings. Not only are such ratings subjective, but the ratings also involved an averaging of one's perceived symptom intensity throughout the preceding week. It is possible that the results may be different when peak symptom intensity is considered, as opposed to average symptom intensity throughout the week. Although capturing continuous psychophysiological measurements would be ideal, there is still value in investigating self-reported symptoms, as these are the symptoms (subjectively biased or not) which patients are most likely to describe to clinicians at routine assessments. Our findings, then, provide insights that may assist clinicians in discriminating different disorder groups when assessing their patients. Relying on a single, well-established selfreport questionnaire also ensured that each symptom was rated on the same scale. It also afforded assessment of a broader range of autonomic symptoms than psychophysiological measures would 
allow. Second, we were unable to capture the full range of autonomic symptoms. For example, there were no items pertaining to sweating, which is an important symptom of autonomic arousal. Nonetheless, we believe that the SCL-90R items otherwise provided a good overall coverage of symptoms for the purpose of the present study. Third, our assessment of AAS was limited to the preceding 7-days precluding any conclusions about trait-like reporting of AAS. Finally, individuals with PTSD are not treated at our facilities, precluding the investigation of the place of AAS in PTSD.

The limitations of this study notwithstanding, the present findings suggest that the selfreported AAS which we assessed characterise most anxiety disorders, with these symptoms being less prominent in OCD. These findings have significant clinical implications: First, standard clinical assessment of such self-reported symptoms is insufficient to distinguish between certain anxiety disorders (i.e., panic disorder, GAD and social anxiety disorder). Further, to the extent that anxiety disorders are defined by high levels of self-reported AAS, our findings support the conceptualisation of OCD as distinct from anxiety disorders. Our results also provide the first evidence that selfreported AAS do not appear to differ between separate OCD symptom dimensions. 


\section{References}

American Psychiatric Association, 2013. Diagnostic and Statistical Manual of Mental Disorders, Fifth Edition (DSM-5). Washington, DC: American Psychiatric Association.

Andrews, G., Charney, D. S., \& Sirovatka, P. J., 2009. Stress induced and fear circuitry disorders: Refining the research agenda for DSM-V. Arlington, VA: American Psychiatric Association. Berle, D., Starcevic, V., Brakoulias, V., Sammut, P., Milicevic, D., Hannan, A., \& Moses, K., 2012. Disgust propensity in obsessive-compulsive disorder: Cross-sectional and prospective relationships. J Behav Ther Exp Psychiatry, 43, 656-663. doi: 10.1016/j.jbtep.2011.09.002

Briggs, A. C., Stretch, D. D., \& Brandon, S., 1993. Subtyping of panic disorder by symptom profile. Br J Psychiatry, 163, 201-209. doi: 10.1192/bjp.163.2.201

Brown, T. A., Chorpita, B. F., \& Barlow, D. H., 1998. Structural relationships among dimensions of the DSM-IV anxiety and mood disorders and dimensions of negative affect, positive affect, and autonomic arousal. J Abnorm Psychol, 107, 179-192. doi: 10.1037/0021-843X.107.2.179

Brown, T. A., \& McNiff, J., 2009. Specificity of autonomic arousal to DSM-IV panic disorder and postraumatic disorder. Behav ResTher, 47, 497-493. doi:10.1016/j.brat.2009.02.016

Clark, D. A., \& Watson, D., 1991. Tripartite model of anxiety and depression: Psychometric evidence and taxonomic implications. J Abnorm Child Psychol, 100, 316-336. doi:10.1037/0021843X.100.3.316

Collins, L. M., \& Lanza, S. T., 2010. Latent class and latent transition analysis: With applications in the social behavioral, and health sciences. Hoboken, NJ: Wiley.

Derogatis, L. R., 1994. SCL-90R administration, scoring and procedures manual, Third ed. Minneapolis, MN: NCS Pearson.

Forbes, D., Lockwood, E., Elhai, J. D., Creamer, M., O'Donnell, M., Bryant, R., . . Silove, D., 2011. An examination of the structure of posttraumatic stress disorder in relation to the anxiety and depressive disorders. J Affect Disord, 132, 165-172. doi:10.1016/j.jad.2011.02.011 
Freire, R. C., Perna, G., \& Nardi, A. E., 2010. Panic disorder respiratory subtype: Psychopathology, laboratory challenge tests, and response to treatment. Harv Rev Psychiatry, 18, 220-229. doi:10.3109/10673229.2010.493744

Gerlach, A. L., Wilhelm, F. H., Gruber, K., \& Roth, W. T., 2001. Blushing and physiological arousability in social phobia. J Abnorm Psychol, 110, 247-258. doi: 10.1037/0021-843X.110.2.247

Hofmann, S. G., \& Spiegal, D. A., 1999. Panic control treatment and its applications. Journal of Psychotherapy Practice and Research, 8, 3-11.

Jack, M. S., Heimberg, R. G., \& Mennin, D. S., 1999. Situational panic attacks: impact on distress and impairment among patients with social phobia. Depress Anxiety, 10, 112-118. doi: 10.1002/(SICI)1520-6394(1999)10:3<112::AID-DA4>3.0.CO;2-U

Mancini, F., \& Gangemi, A., 2004. Fear of guilt from behaving irresponsibly in obsessive-compulsive disorder. J Behav Ther Exp Psychiatry, 35, 109-120. doi:10.1016/j.jbtep.2004.04.003

Mancini, F., Gangemi, A., Perdighe, C., \& Marini, C., 2008. Not just right experience: is it influenced by feelings of guilt? J Behav Ther Exp Psychiatry, 39, 162-176. doi:10.1016/j.jbtep.2007.02.002

Meyer, T. J., Miller, M. L., Metzger, R. L., \& Borkovec, T. D., 1990. Development and validation of the Penn State Worry Questionnaire. Behav Res Ther, 28, 487-495. doi: 10.1016/00057967(90)90135-6

Prenoveau, J. M., Zinbarg, R. E., Craske, M. G., Mineka, S., Griffith, J. W., \& Epstein, A. M., 2010. Testing a hierarchical model of anxiety and depression in adolescents: a tri-level model. J Anxiety Disord, 24, 334-344. doi:10.1016/j.janxdis.2010.01.006

Pruneti, C. A., Lento, R. M., Fante, C., Carrozzo, E., \& Fontana, F., 2010. Autonomic arousal and differential diagnosis in clinical psychology and psychopathology. Giornale Italiano Di Psicopatologia, 16, 43-52. 
Raines, A. M., Allan, N. P., Oglesby, M. E., Short, N. A., \& Schmidt, N. B., 2015. Examination of the relations between obsessive-compulsive symptom dimensions and fear and distress disorder symptoms. J Affect Disord, 183, 253-257. doi:10.1016/j.jad.2015.05.013

Rickels, K., \& Rynn, M.A., 2001. What is generalized anxiety disorder? J Clin Psychiatry, 62, 4-12.

Roberson-Nay, R., Latendresse, S. J., \& Kendler, K. S., 2012. A latent class approach to the external validation of respiratory and non-respiratory panic subtypes. Psychol Med, 42, 461-474. doi:10.1017/S0033291711001425

Shear, M. K., Brown, T. A., Barlow, D. H., Money, R., Sholomskas, D. E., Woods, S. W., . . Papp, L. A., 1997. Multicenter collaborative panic disorder severity scale. Am J Psychiatry, 154, 15711575. doi:10.1176/ajp.154.11.1571

Sheehan, D., Janavs, J., Baker, R., Harnett-Sheehan, K., Knapp, E., Sheehan, M., . . Lepine, J. P., 1999. The MINI Interntational Neuropsychiatric Interview (M.I.N.I). English version 5.0.0. DSM-IV. Journal of Clinical Psychiatry, 60, 39-62.

Stein, M.B., Roy-Byrne, P.P., Craske, M.G., ... Sherbourne, C. D., 2005. Functional impact and health utility of anxiety disorders in primary care outpatients. Med Care, 43, 1164-1170.

Sullivan, G., Craske, M. G., Sherbourne, C., Edlund, M. J., Rose, R. D., Golinelli, D., . . Roy-Byrne, P. P., 2007. Design of the Coordinated Anxiety Learning and Management (CALM) study: innovations in collaborative care for anxiety disorders. Gen Hosp Psychiatry, 29, 379-387. doi:10.1016/j.genhosppsych.2007.04.005

Thordarson, D. S., Radomsky, A. S., Rachman, S., Shafran, R., Sawchuk, C. N., \& Ralph Hakstian, A., 2004. The Vancouver Obsessional Compulsive Inventory (VOCI). Behav Res Ther, 42, 12891314. doi:10.1016/j.brat.2003.08.007

Watson, D., 2005. Rethinking the mood and anxiety disorders: A quantitative hierarchical model for DSM-V. J Abnorm Psychol, 114, 522-536. doi: 10.1037/0021-843X.114.4.522 
World Health Organisation, 1992. The ICD-10 (International Classification of Diseases) Classification of Mental and Behavioural Disorders: Clinical Descriptions and Diagnostic Guidelines. Geneva: World Health Organization. 
Table 1. Incremental fit statistics and classification accuracy for latent class analysis $(N=459)$.

\begin{tabular}{ccccccc}
\hline $\begin{array}{c}\text { Number } \\
\text { of } \\
\text { classes }\end{array}$ & LogLikelihood & AIC & BIC & Entropy & LMRLRT & $\begin{array}{c}\text { LMRLRT } p \text { - } \\
\text { value }\end{array}$ \\
\hline 1 & -6003.9 & 12081.9 & 12234.6 & - & - & - \\
2 & -5366.7 & 10883.5 & 11193.2 & 0.91 & -6003.93 & $<0.001$ \\
3 & -5226.1 & 10678.3 & 11144.9 & 0.88 & -5366.74 & 0.02 \\
4 & -5149.0 & 10599.9 & 11223.4 & 0.84 & -5226.13 & 0.27 \\
5 & -5086.7 & 10551.4 & 11331.8 & 0.88 & -5148.95 & 0.10 \\
6 & -5048.5 & 10551.0 & 11488.3 & 0.90 & -5086.71 & 0.76 \\
\hline
\end{tabular}

$\mathrm{AIC}=$ Akaike Information Criterion; $\mathrm{BIC}=$ Bayesian Information Criterion;

LMRLRT = Low-Mendell Rubin Likelihood ratio test. 
Table 2. Logistic regression analysis predicting membership in the high autonomic arousal symptom class.

\begin{tabular}{lcccc}
\hline Predictor variables & $\begin{array}{c}\text { Unstandardized } \\
\mathrm{B}(\mathrm{SE})\end{array}$ & Wald test (z-ratio) & Exp(B) & $p$ \\
\hline (Constant) & 2.620 & 0.53 & 13.74 & \\
Gender & -0.092 & 0.17 & 0.99 & 0.68 \\
Age & 0.016 & 4.36 & 0.99 & 0.04 \\
Major depressive disorder & 0.399 & 2.40 & 0.67 & 0.12 \\
Panic disorder & 1.581 & 39.39 & 0.21 & $<0.001$ \\
Social anxiety disorder & 0.521 & 4.10 & 0.59 & 0.04 \\
Obsessive-compulsive disorder & 0.336 & 1.86 & 0.71 & 0.17 \\
Generalized anxiety disorder & 0.626 & 8.37 & 0.54 & 0.004 \\
Specific phobia & 0.023 & 0.008 & 1.02 & 0.93 \\
\hline
\end{tabular}


Table 3. Logistic regression analysis of the $\mathrm{VOCl}$ subscales predicting membership in the high autonomic arousal symptom class.

\begin{tabular}{lcccc}
\hline Vocl subscale & $\begin{array}{c}\text { Unstandardised } \\
\mathrm{B}(\mathrm{SE})\end{array}$ & $\begin{array}{c}\text { Wald test } \\
\text { (z-ratio) }\end{array}$ & Exp(B) & $p$ \\
\hline (Constant) & $0.56(0.34)$ & 2.73 & 1.76 & 0.10 \\
Contamination & $-0.01(0.01)$ & 1.13 & 0.99 & 0.29 \\
Checking & $-0.01(0.02)$ & 0.36 & 0.99 & 0.55 \\
Obsessions & $0.01(0.02)$ & 0.22 & 1.01 & 0.64 \\
Hoarding & $0.003(0.02)$ & 0.02 & 1.00 & 0.89 \\
Not just right experiences & $0.03(0.02)$ & 1.33 & 1.03 & 0.25 \\
Indecisiveness & $-0.04(0.04)$ & 1.27 & 0.96 & 0.26 \\
\hline
\end{tabular}

$\mathrm{VOCl}=$ Vancouver Obsessive Compulsive Inventory. 
Figure 1. Conditional response probability plots for the two class solution at each level of item endorsement.
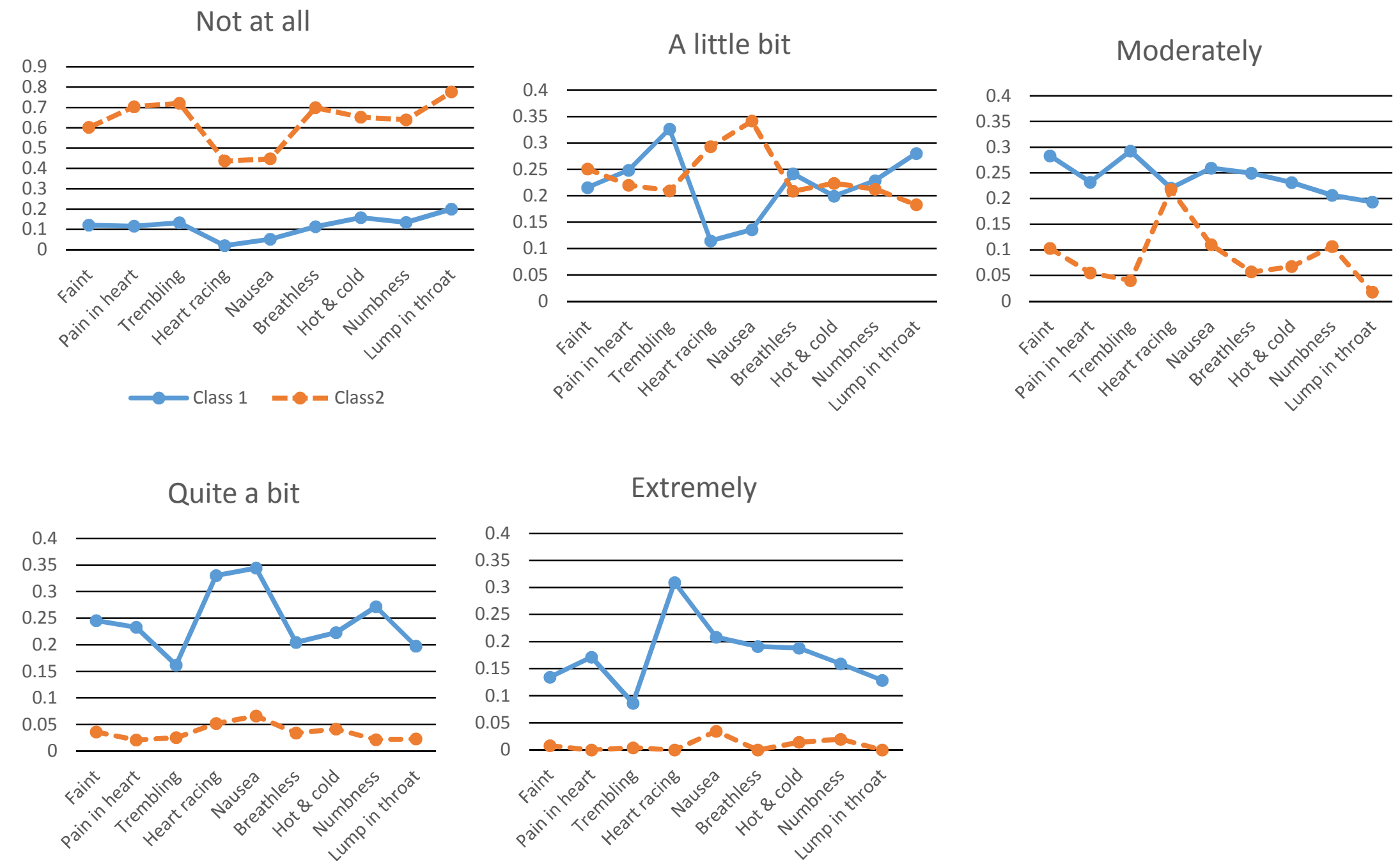